\section{Kinetic Analysis of Plasma Radioactivity after Oral Ingestion of Radiocalcium}

THE absorption of calcium from the gastro-intestinal tract is one of the chief regulators of calcium metabolism and is known to be disturbed in a variety of clinical disorders. Despite its importance, however, no rapid, accurate procedure is available for its measurement, which normally requires the estimation of dietary and faecal calcium over a period of weeks. Several workers have therefore sought to establish an isotopic procedure which would enable calcium absorption to be calculated from the plasma radioactivity ${ }^{1,2}$ or residual faecal activity ${ }^{3,4}$ following an oral dose of radioactive calcium. It has been found, however, that although plasma radioactivity is inversely related to faecal recovery, it is not, by itself, a reliable measure of calcium absorption ${ }^{3,4}$. This is not surprising in view of the fact that the concentration of tracer at any given time after administration must be determined not only by the rate of its absorption into plasma, but also by the rate of its removal, and some workers have used double isotope techniques to overcome this difficulty ${ }^{5}$.

In seeking to improve the diagnostic value and physiological meaning of plasma radioactivity measurements after an oral dose of radiocalcium, we postulate that both the absorption and removal processes can be described by simple exponential functions ${ }^{6}$ and we have shown that diagnostic precision can be improved, particularly in malabsorption states, by a simple approximation which

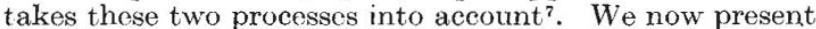
a development of this model and a more accurate mathematical expression which permits the calculation of the rate of calcium absorption without the necessity for faecal collection.

The model is shown in Fig. 1. Labelled calcium is assumed to be absorbed from the small intestine at a rate $\alpha$, proportional to the residual concentration. (The alternative assumption- that a constant amount of calcium is absorbed per unit time-has been tested and found not to fit the observed data.) The labelled calcium Inixes with the calcium in the plasma and extracellular fluid $(A)$ from which it is removed into bone and excreted into the urine and faeces. In the first few hours, most of this removal (probably about 95 per cent) occurs into bone by exchange processes which can be expressed as a power function ${ }^{8}$, but for ease of calculation they can be approximated to a double exponential over a strictly limited period. We have termed the fractional rate of radioactive calcium entry into bone by this process $\beta$ and the return rate $\gamma$.

The mathematical solution of this model has the form

$$
f=\frac{\alpha-\gamma}{\beta+\gamma-\alpha} \mathrm{e}^{-\alpha t}-\frac{\alpha \beta}{(\beta+\gamma)(\beta+\gamma-\alpha)} \mathrm{e}^{-(\beta+\gamma) t}+\frac{\gamma}{\beta+\gamma}
$$

where $f$ is the fraction of the dose circulating in the plasma and extracollular fluid (activity/l. $\times 15$ per cent of bodyweight) at any time $t$ after the dose.

This expression has been fitted to the mean plasma activity values reported by Avioli et al. ${ }^{2}$ in subjects with

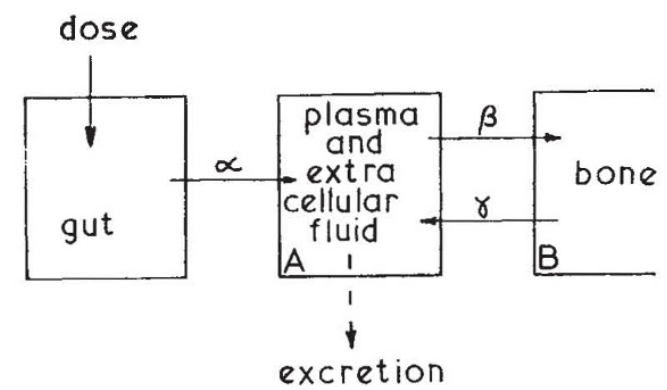

Fig. 1. Suggested model for the calculation of absorption of radiocalcium following a single oral dose. $A$, Plasma and extracellular tluid;
$B$, bone.

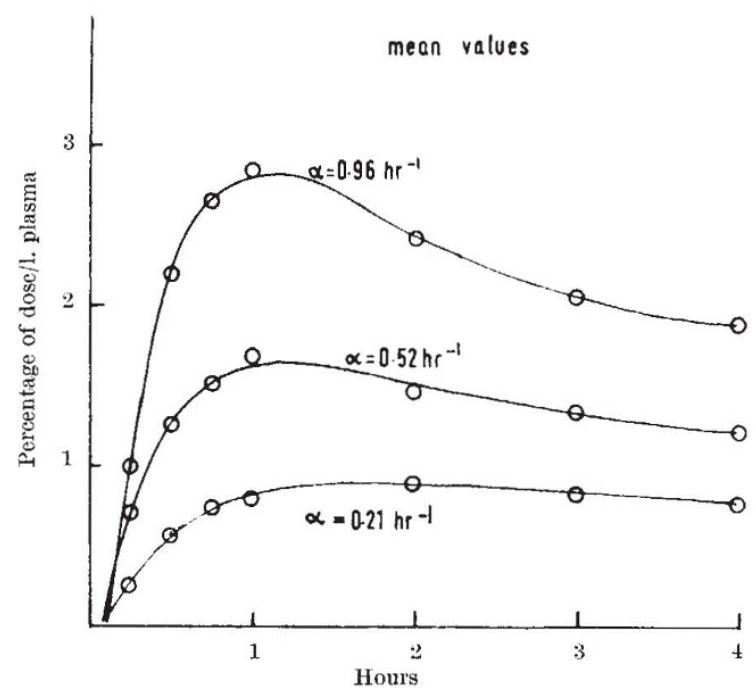

Fig. 2. Curves calculated from the mean data of A violi $e t a l .^{2}$ in hyperabsorbers, normals and malabsorbers.

known high, normal and low calcium absorption. The calculated curves are plotted with the data points in Fig. 2. The excellent agreement between the data and the theoretical curves suggests that our model is an adequate one. Individual $\alpha, \beta$ and $\gamma$ values have also been calculated for all the data, and the means in the three groups are shown in Table 1 . The mean normal $\alpha$ (rate of absorption) is $0.62 \mathrm{~h}^{-1}$ and the corresponding values for the high and low absorbers are 1.05 and 0.23 respectively. The mean $\beta$ (removal into bone) and $\gamma$ (return from bone) values do not differ between the three groups except that the $\gamma$ value is low in malabsorption. This indicates that the exchangeable ealcium pool in the bone is much larger in these cases than in the normals and high absorbers and suggests that some of these patients may be developing or have developed osteomalacia, in which condition pool size and tumover are known to be high? .

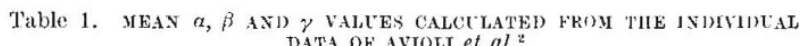

$\begin{array}{cccc}\text { Group } & \alpha \mathrm{h}^{-1} & \beta \mathrm{h}^{-1} & \gamma \mathrm{H}^{-1} \\ \text { low absorbers } & 0 \cdot 23 \pm 0 \cdot 07 & 2 \cdot 12+0 \cdot 40 & 0 \cdot 08 \pm 0 \cdot 06 \\ \text { Normal } & 0 \cdot 62 \pm 0 \cdot 20 & 2 \cdot 51 \pm 0 \cdot \frac{7}{7} & 0 \cdot 31 \pm 0 \cdot 15 \\ \text { High absorbers } & 1 \cdot 05 \pm 0 \cdot 40 & 1 \cdot 95 \pm 0 \cdot 75 & 0 \cdot 39 \pm 0 \cdot 19\end{array}$

We suggest that this form of calculation will probably permit fairly accurate measurement of calcium absorption rates at low carrier levels from simple analysis of plasma radioactivity curves, and that it may be applicable to the absorption of substances other than calcium.

D. H. MARSHALL

MRC Enviroumental Radiation Research Unit,

Department of Medical Physics,

The General Infirmary, Leeds 1.

B. E. C. NORnIN

MRC Mineral Metabolism Research Unit,

The General Infirmary, Leeds I.

Received December 12, 1968; revised March 31, 1969.

${ }^{1}$ Bhandarkar, s. 1)., Bluhm, M. M., MacGregor, J., and Fordin, B. L. (‘, Brit. Med. J.,2,1534(1961).

2 Avioli, T. V., MeDonald, J. E., Singer, R. A., anl Henneman, P. M.. J. Clin. Invest., 44, 128 (1965).

${ }^{3}$ Kinney, V. R., Tauxe, W. N., and Dearing, W. H., J. Lab. Clin. Metl., 66, $187^{(1965)}$.

4 Jaworski, Z. F., Brown, E. M., Fedoruk, S., and Scitz, II., Feen Eingl. J. Med., 269, 187 (1963).

${ }^{5}$ DeGrazia, J. A., Tvanovich, P., Fellows, H., and Rich, C. J., J. Lab. Clin. Ired., 66. $822(1965)$

' Nordin, B. E. (:., (fastroenterol., 54, 294 (1968),

Nordin, B. E. C., Young, M. M., Oxby, (. B., and Bulusu, L., Clin. Siri., 35, $177(1968)$.

${ }^{*}$ Burkinshaw, L., Marshall, D. H., Nordin, B. E. C., Oxby, ('. B., Spiers, F. W., and Y̌oung, M. M., Nature, 222, 146 (1969).

Yordin, B. E. C., and Smith, D. A., LOsteomalacie (edit. by Hioco, J. J.), 379 (Masson, Paris, 1967) 